\title{
The emission of sound by statistically homogeneous bubble layers
}

\author{
L. van WIJNGAARDEN and J. BUIST* \\ University of Twente, P.O Box 217, 7500 AE Enschede, The Netherlands; * present address: Ultracentrifuge \\ Nederland, P.O. Box 158, 7600 AD Almelo, The Netherlands
}

\begin{abstract}
This paper is concerned with the flow of a bubbly fluid along a wavy wall, which is one Fourier component of a linearized hydrofoil. The bubbles are dispersed, not throughout the whole of the liquid, but only over a certain distance from the wall, as occurs in practice with cavitation bubbles. Outside the bubbly regime there is pure liquid.

The interface between the bubbly fluid and pure liquid fluctuates for various reasons. One of these is the relative motion between bubbles and liquid. This is considered here in detail. A calculation is made of the sound emitted by the bubbly layer into pure liquid as a result of this stochastic motion of the interface.
\end{abstract}

\section{Introduction}

Cavitating ship propellers emit sound. This may reach in the region around $1 \mathrm{KHz}$ an intensity of $150 \mathrm{~dB}$ in the far field spectrum. The dangers associated with such a strong sound emission are obvious for navy vessels, but also with merchant ships the strong sound emission causes great discomfort inboard. The spectral distribution of the emitted sound looks like that sketched in Fig. 1, taken from the thesis by Buist [1]. Such distributions can be measured on model scale. It is, at the present time, not possible to predict from such measurements what the full scale emission is, in magnitude and in spectral distribution. A study on cavitation sound prediction is being made by us in cooperation with the Maritime Research Institute in the Netherlands (MARIN).

It has been known for a long time that the sound stems from the collapse of cavitation bubbles. Recently, however, evidence was gained that only at high frequencies does the sound come from individual bubbles, while at lower frequencies interaction between bubbles is important.

The work by Omta [2] and Buist [1] showed in particular, that a broad plateau at $1 \mathrm{KHz}$ is generated by clouds of collapsing bubbles. At MARIN experiments were carried out in this connection. A hydrofoil (for a detailed description see Buist [1]) was positioned in a cavitation tunnel. Small gas bubbles were injected in the flow adjacent to the hydrofoil. In this way a bubbly flow passed closely near the foil. Under influence of the pressure distribution associated with the flow along the hydrofoil, the bubbles grew and collapsed. ${ }^{\dagger}$ Pressures in the water flow, resulting from this interaction between the bubbly layer and the outer (pure liquid) flow, were measured. Bubbles could, at the injection site, be produced intermittently or continually. In both cases the measured sound intensity, as derived from the pressure fluctuations, agreed well with the theory in Omta [2] and Buist [1]. In these theories the hydrodynamics of collapsing clouds of bubbles is considered. For the phenomena inside a cloud, equations are used which were derived in Biesheuvel \& van Wijngaarden

\footnotetext{
${ }^{+}$In practice bubbles contain both gas and vapour. The difference in behaviour with gas filled bubbles is quantitative
} rather than qualitative. 


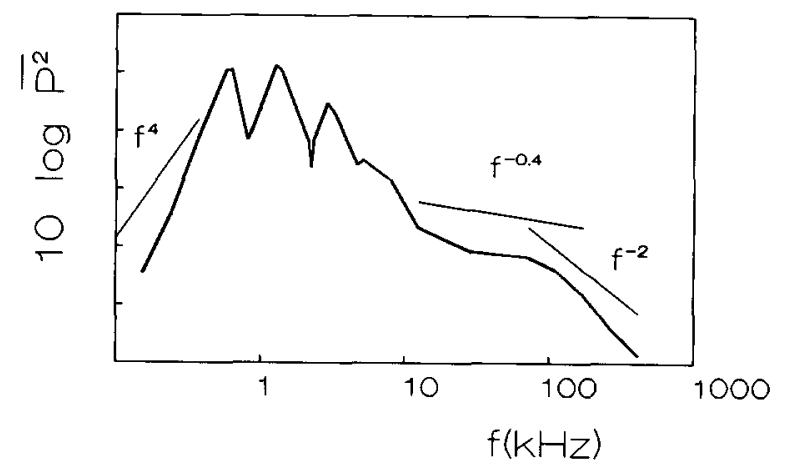

Fig. 1. Schematical representation of the spectral distribution of the sound emitted by a cavitating ship propeller. From Buist [1].

[3]. These equations are established by averaging on the mesoscale. The latter is a scale in between the microscale (in the case of a bubble cloud represented by the inter bubble distance) and the macroscale (for example the chord length of the hydrofoil). Such average equations predict a steady flow, both in the bubble layer and in the outer flow, when external parameters as incidence, rate of injection of bubbles and incoming flow velocity, are kept constant.

Such a flow, by its steady nature, does not emit sound. Nevertheless, sound emission is observed. This has two causes. Firstly, bubbles cluster together and form clouds, which is an unsteady process. Secondly, fluctuations around the average quantities contribute to sound emission. These fluctuations are suppressed by the averaging process. It is important to estimate the contribution by the fluctuations, because, if they are important, the use of average equations would be dubious or would, at the least, not provide a sufficiently complete description of the generated sound intensity. In the present paper our attention is focused on the sound emission by fluctuations. In a bubbly flow various fluctuations may occur. Buist [1], see also Buist [4], considers fluctuations of the local void fraction caused by fluctuations of the pressure inside the bubbles. He concludes that the sound intensity produced by these can be estimated to be $90 \mathrm{~dB}$, which is considerably smaller than the observed sound intensity of cavitating flows which is as mentioned earlier, $150 \mathrm{~dB}$.

Another type of fluctuations mentioned by Buist [1], but not taken further into consideration, is caused by relative motion between bubbles and liquid. Relative motion in a bubbly flow occurs whenever there are accelerative forces. In vertical pipe flow, for example, this is buoyancy. In the case of the flow of a bubbly fluid along a hydrofoil it is the pressure gradient associated with the curvature of the foil. This works on both the gas and on the liquid, but because of the different inertia, both phases obtain different velocities under the same pressure gradient. The relative motion causes fluctuating velocities in the bubbly part of the fluid and therefore also of the interface between this and pure liquid outside the bubbly layer. The fluctuations of the interface, in turn, lead to sound emission in the pure liquid. Our aim is to calculate the intensity of this. We don't take a complete hydrofoil but consider instead an infinitely long wavy wall of wavelength $\lambda$ only. The wavy wall is a well known concept in aerodynamics because, within the context of linear theory, the flow along a thin hydrofoil can be obtained from the analysis of one wavelength by Fourier synthesis.

The flow configuration is sketched in Fig. 2: along a wavy wall the height of which is in a two-dimensional $x, y$ frame given as the real part of 


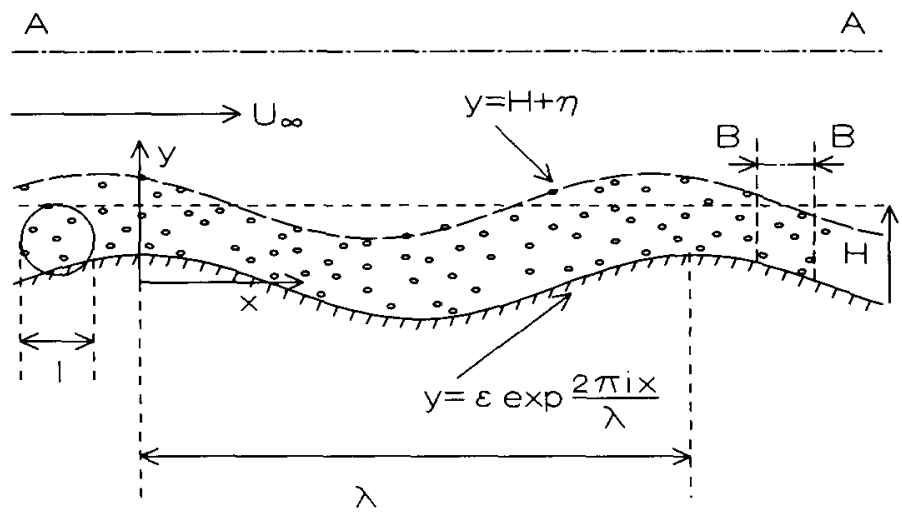

Fig. 2. Flow of a bubble/liquid mixture along a wavy wall. Between the wall and the height $y=H+\eta$ is a mixture of air bubbles and water. For $y \geqslant H+\eta$, there is pure water.

$$
y=\varepsilon \exp \left(\frac{2 \pi \mathrm{i} x}{\lambda}\right)
$$

is a flow in part consisting of a bubbly liquid, and pure liquid in the remainder. The average interface is at $y=H$, the local interface at $y=H+\eta(x, t)$. The undisturbed velocity is $U_{\infty}$ in both parts, independent of $x, y$ and the time $t$. In the bubbly flow the macroscale is the wavelength $\lambda$, the microscale is the mean distance between bubbles. When the number density of the bubbles is indicated $n$, this is proportional to $n^{-1 / 3}$. In order that averages be meaningful, we must require

$$
n^{-1 / 3} \ll \lambda
$$

The averaging is done over a region with linear dimension $l$, say, small with respect to $\lambda$ but large with respect to $n^{-1 / 3}$, as symbolically indicated in Fig. 2. The part of the displacement $\eta$ which is due to the mean flow (on the scale $l$ ) and which we denote $\eta_{1}(x)$ is steady. The part which is due to the fluctuations is denoted $\eta_{2}(x, t)$ and interests us here.

Flow of a bubbly liquid along a wavy wall occupying the upper half plane $y \geqslant 0$, has been analysed by Agostino et al. [5] some years ago. They considered flows in which relative motion between phases can be neglected and calculated for such flows distributions of void fraction, velocity and pressure. Buist [1] extended the work by Agostino et al. [5], to the situation of Fig. 2. In the next section we shall use Buist's analysis to determine the relative motion between phases in situation of Fig. 2. Then, in Section 3, we shall investigate the influence of relative motion on the fluctuations of the interface, $\eta_{2}(x, t)$. Finally, in Section 4 , we shall relate the sound energy emitted through a plane $A-A$, in Fig. 2 , to the fluctuations $\eta_{2}(x, t)$ of the interface.

\section{Mean quantities including relative motion, in bubbly layer}

We start with the introduction of some parameters, characterising the bubbly mixture contained, in Fig. 2, between the wavy wall and pure liquid. Let the undisturbed radius of each bubble be $a_{0}$ and $a(x, y)$ in the general case. Together with the number density $n, a$ 
defines the void fraction $\alpha$

$$
\alpha=\frac{4}{3} \pi n a^{3}
$$

Since $\alpha \sim\left(a / n^{-1 / 3}\right)^{3}$, the void fraction is small when the bubble radius is small with respect to the inter bubble distance. This is always the case in cavitating flows and we may therefore in the following regard $\alpha$ as a small quantity, $\alpha \ll 1$. Let the (constant) liquid density be $\rho_{0}$ and let $p_{0}$ be the pressure in the undisturbed state. It is well known (see e.g. Van Wijngaarden [6]) that a bubbly suspension has a sound velocity $c_{m}$ which is considerably lower than the sound velocity either in liquid or in gas, and given, for infinite wavelength, by

$$
c_{m}^{2}=\frac{\gamma p_{0}}{\rho_{0} \alpha_{0}}
$$

where $\gamma$ is the ratio of specific heats of the gas in the bubbles. At finite wavelength the pressure inside the bubbles is no longer equal to the pressure in the fluid adjacent to the bubble, which leads to dispersion. In the case of Fig. 2 this depends on the frequency $k U_{x}$, where

$$
k=2 \pi / \lambda
$$

as compared with the natural frequency

$$
\omega_{b}=a_{0}^{-1}\left\{3 \gamma p_{0} / \rho_{0}\right\}^{1 / 2},
$$

of the bubble.

For $\omega_{b} \gg k U_{\infty}$ we have (2.2) whereas for wavelengths $k$ such that $k U_{\infty}$ is comparable with $\omega_{b}$, the modified sound speed $c_{m}$ holds, given by

$$
\tilde{c}_{m}^{-2}=c_{m}^{-2}\left\{\omega_{b}^{2} /\left(\omega_{b}^{2}-k^{2} U_{\infty}^{2}\right)\right\}
$$

In our present application it is highly unlikely that $k U_{\infty}$ would ever become close to $\omega_{b}$. With $a_{0} \simeq 10^{-3} \mathrm{~m}, \omega_{b} \sim 10^{4} \mathrm{~s}^{-1}$. Since we are under cavitating circumstances, $\rho_{0} U_{\infty}^{2} \sim 10^{5} P_{a}$, which with $\rho_{0}=10^{3} \mathrm{~kg} / \mathrm{m}^{3}$ means $U_{\infty} \sim 10 \mathrm{~m} / \mathrm{s}$. Then $k U_{\infty}=\omega_{b}$ for wavelengths of a few millimeter. With hydrofoils such small wavelengths do not occur, perhaps approximately at the very leading edge. Elsewhere $\lambda$ is of the order of the chord length and in consequence $k U_{\infty} \ll \omega_{b}$. We shall therefore in the following neglect $k U_{\infty}$ with respect to $\omega_{b}$. Then, a Mach number $M$ follows naturally as

$$
M=U_{\infty} / c_{m}
$$

In the present application $M$ will be low. For example, take $\alpha=5 \%, p_{0}=10^{5} P_{a}, \rho_{0}$ as before and $\gamma=1.4$. With, as above, $U_{\infty}=10 \mathrm{~m} / \mathrm{s}$, we obtain $M=0.1$.

Buist [1] derived expressions for pressure, velocity and void fraction for the configuration of Fig. 2, using in the region occupied by the bubbly suspension, the averaged equations given in Biesheuvel and van Wijngaarden [3]. From the analysis in Buist [1], it follows that 
the pressure perturbation is the real part of

$$
\begin{aligned}
p_{m}-p_{01}= & -\frac{\rho_{0} \varepsilon k U_{x}^{2} \exp (\mathrm{i} k x)}{\left(1-M^{2}\right)^{1 / 2}}\left[\exp \left\{-\left(1-M^{2}\right)^{1 / 2} k y\right\}\right. \\
& \left.+\frac{\left\{\left(1-\alpha_{0}\right)\left(1-M^{2}\right)^{1 / 2}-\left(1-2 \alpha_{0}\right)\right\}\left(\exp \left\{\left(1-M^{2}\right)^{1 / 2} k(y-H)\right\}+\exp \left\{-\left(1-M^{2}\right)^{1 / 2} k(y+H)\right\}\right)}{2 \cosh \left\{\left(1-M^{2}\right)^{1 / 2} k H\right\}+2\left(1-M^{2}\right)^{1 / 2} \sinh \left\{\left(1-M^{2}\right)^{1 / 2} k H\right\}}\right]
\end{aligned}
$$

This is a rather formidable expression, which for our purpose can be simplified considerably, since we are interested in low values of both $M$ and $\alpha$. To see the contributions to $p_{m}-p_{0}$ of various effects, let us take first $M=0$ and $H \rightarrow \infty$. Then

$$
p_{m}-p_{0}=-\rho_{0} \varepsilon k U_{\infty}^{2} \exp (\mathrm{i} k x) \exp (-k y)
$$

which satisfies Laplace's equation and is $-\rho_{0} U_{\infty}$ times the velocity associated with potential flow along the linearized wavy wall. Next take $H \rightarrow \infty$ and $M \neq 0$. Then the second term in the square brackets on the right-hand side of (2.7) vanishes and the remainder is the pressure distribution of the wavy wall flow of a compressible flow with Mach number $M$, to be found in many textbooks on gas dynamics, e.g. Liepmann \& Roshko [7]. At finite values of $H$, the second term in the square brackets in (2.7) appears to be small at small $M$ and $\alpha_{0}$. Take for example $M=0$ and $y=H$. Then

$$
p_{m}-p_{0}=-\rho_{0} \varepsilon k U_{\infty}^{2} \exp (i k x) \exp (-k H)\left[1+\frac{1}{2} \alpha_{0}(1+\exp (-2 k H))\right]
$$

We see that the contribution of the second term is of order $\alpha_{0}$. In other words, the pressure distribution in the bubbly layer deviates at zero Mach number to order $\alpha_{0}$ from the expression valid in pure liquid. This remains the case at small Mach numbers when $M^{2}$ is of order $\alpha_{0}$, as can be readily verified.

We shall therefore base our calculation of the relative velocity between liquid and bubbles on the pressure distribution given in (2.7), without the second term in the square brackets,

$$
\begin{aligned}
& p_{m}-p_{0}=-\frac{\rho_{0} \varepsilon k U_{\infty}^{2}}{\left(1-M^{2}\right)^{1 / 2}} \exp (\mathrm{i} k x) \exp \left\{-\left(1-M^{2}\right)^{1 / 2} k y\right\} \\
& 0 \leqslant y \leqslant H .
\end{aligned}
$$

The velocity perturbations $u-U_{\infty}$ and $v$, in $x$ and $y$ direction respectively associated with this pressure perturbation, are (real parts being implied)

$$
\begin{aligned}
& u-U_{\infty}=\frac{\varepsilon k U_{\infty}}{\left(1-M^{2}\right)^{1 / 2}} \exp (\mathrm{i} k x) \exp \left\{-\left(1-M^{2}\right)^{1 / 2} k y\right\}, \\
& v=\varepsilon k \mathrm{i} U_{\infty} \exp (\mathrm{i} k x) \exp \left\{-\left(1-M^{2}\right)^{1 / 2} k y\right\}
\end{aligned}
$$

Relations (2.9) and (2.8) show the well known facts that the $x$-component of the velocity is in phase with the wall elevation, the pressure in anti-phase.

The pressure gradient, following from (2.8) produces relative motion between bubbles and liquid. Let us first assume that this is dominated by inertia forces rather than by viscous 
forces. Denoting the velocity in the liquid $\mathbf{U}(u, v)$ and of the bubbles $\mathbf{V}\left(V_{x}, V_{y}\right)$, we have, $D / D t$ being a material derivative following the bubbles (Biesheuvel and van Wijngaarden [3]),

$$
\frac{D}{D t} m(\mathbf{V}-\mathbf{U})=-V \nabla p_{m}=\rho_{0} V \frac{D \mathbf{U}}{D t}
$$

Here $m$ is the virtual mass of a bubble, which has for spherical bubbles the value $1 / 2 \rho_{0} V, V$ being the volume of one bubble. The above relation gives, for spherical bubbles, the result that the bubbles move at three times the liquid velocity. Incidentally, we note that using a material derivative following the liquid where $\mathbf{U}$ is concerned, which is sometimes advocated in the multiphase literature, would not make any difference because of the linearisation around $U_{\infty}$.

The relative velocity of the bubbles with respect to the liquid has therefore the components

$$
\begin{aligned}
& V_{x}-u=\frac{2 \varepsilon k U_{\infty}}{\left(1-M^{2}\right)^{1 / 2}} \exp (\mathrm{i} k x) \exp \left\{-\left(1-M^{2}\right)^{1 / 2} k y\right\} . \\
& V_{y}-v=2 \mathrm{i} \varepsilon k U_{\infty} \exp (\mathrm{i} k x) \exp \left\{-\left(1-M^{2}\right)^{1 / 2} k y\right\} .
\end{aligned}
$$

Of course, frictional forces affect the relative motion. The time which it takes viscous forces to influence the motion, the so-called relaxation time, $\sigma$, say, is the product of the virtual mass $m$ and the mobility $(12 \pi \mu a)^{-1}$, or for a sphere

$$
\sigma=a_{0}^{2} / 18 \nu
$$

where $\mu$ and $\nu$ are the dynamic and kinematic viscosities of the liquid respectively. With $a \simeq 10^{-3} \mathrm{~m}$ and water as liquid, for which $\nu=10^{-6} \mathrm{~m}^{2} / \mathrm{s}, \sigma$ is of the order of $10^{-1}-10^{0}$. The characteristic time for changes in the velocity is $\lambda / U_{\infty}$. The importance of viscous forces depends on whether the ratio between these times, i.e.

$$
\frac{U_{\infty} \sigma}{\lambda}
$$

is small or large. In the first case viscosity is important, in the second case it is not. If we take, like we did earlier, $U_{\infty}=10 \mathrm{~m} / \mathrm{s}$, and if we take $\lambda=10^{-1}$, then with $\sigma=10^{-1}$, this parameter is 10 , with $\sigma=10^{\circ}$ it is $10^{2}$. In practice therefore the parameter $U_{\infty} \sigma / \lambda$ is likely to be large, and in consequence (2.12) and (2.13) are appropriate. Nevertheless, for completeness, we give also the relative velocity in the opposite case in which (2.15) is small. In that case the pressure force represented by the right-hand side of (2.11) is not balanced, like in that equation, by the rate of change of the impulse but rather by the frictional force $12 \pi \mu a(\mathrm{~V}-\mathrm{U})$. This gives the relative velocities, using (2.8)

$$
\begin{aligned}
& V_{x}-u=-\frac{1}{9} \frac{a_{0}^{2}}{\nu} \frac{\varepsilon k^{2} U_{\infty}^{2}}{\left(1-M^{2}\right)^{1 / 2}} \sin (k x) \exp \left\{-\left(1-M^{2}\right)^{1 / 2} y\right\}, \\
& V_{y}-v=-\frac{1}{9} \frac{a_{0}^{2}}{\nu} \varepsilon k^{2} U_{\infty}^{2} \cos (k x) \exp \left\{-\left(1-M^{2}\right)^{1 / 2} y\right\} .
\end{aligned}
$$


Note that the ratio of the magnitude of these velocities to those given in (2.12) and (2.13) is just the parameter described in (2.15), $\sigma$ being given by (2.14).

\section{Sound emitted by fluctuations of $\eta_{2}(x, t)$}

We consider a small portion of a wave length, for example $B-B$ in Fig. 2 . When enlarged this looks as in Fig. 3. We see the interface $\eta_{2}$ under which pass bubbles with relative velocity indicated by the arrows. Far above the interface in the pure liquid is the plane $A-A$, the sound emission through which is of interest here. How is the sound produced? The bubbles are randomly distributed through the liquid. Their motions relative to the liquid (the magnitude of which has been calculated in Section 2), induce a velocity in each point $P^{\prime}$ in the liquid. This also is a randomly varying quantity. In particular we are interested in the velocity at a point $P$ located in the interface. The $y$ component $v$ of this is related to the time derivative of $\eta_{2}$ by

$$
\frac{\partial \eta_{2}}{\partial t}+U_{\infty} \frac{\partial \eta_{2}}{\partial x}=v
$$

In its turn, the motion of the interface can be related to the intensity of the sound emitted through a plane like $A-A$ in Fig. 3. For this it is convenient to follow Buist [1] and to choose a frame moving with the free stream velocity $U_{\infty}$. Such a frame has coordinates $X, Y$ given by

$$
\begin{aligned}
& X=x-U_{\infty} t, \\
& Y=y .
\end{aligned}
$$

In the moving frame $(3.1)$ reduces to

$$
\frac{\partial \eta_{2}}{\partial t}=v
$$

Pictures similar to Fig. 3 are obtained at other locations in the wave of Fig. 2 when the arrows are tilted over an angle corresponding with the local value of $\left(V_{y}-v\right) /\left(V_{x}-u\right)$.

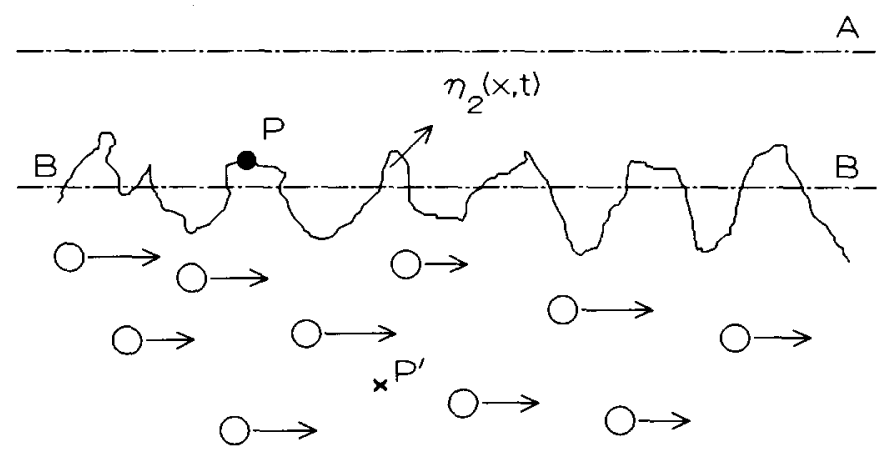

Fig. 3. Fluctuations $\eta_{2}(x, t)$ of the interface between bubbly fluid and pure water, caused by the motion, relative to the water, of bubbles. 
In the region located in Fig. 3 above the bubbly layer, there is motion of pure liquid and when we ignore viscosity this motion is irrotational and has in consequence a velocity potential, $\varphi$, say. The $y$ component of its gradient is the vertical velocity at the interface which, by the kinematic surface condition, must be equal to $v$ in (3.3). Hence we have

$$
\frac{\partial \eta_{2}}{\partial t}=\left(\frac{\partial \varphi}{\partial y}\right)_{y=H} .
$$

The flow potential satisfies the wave equation

$$
\frac{\partial^{2} \varphi}{\partial X^{2}}+\frac{\partial^{2} \varphi}{\partial y^{2}}=\frac{1}{c_{l}^{2}} \frac{\partial^{2} \varphi}{\partial t^{2}},
$$

where $c_{l}$ is the velocity of sound in liquid, $1500 \mathrm{~m} / \mathrm{s}$ for the important case in which the liquid is water. The pressure disturbance $p-p_{0}$ in the liquid is related to $\varphi$ by,

$$
p-p_{0}=-\rho_{0} \frac{\partial \varphi}{\partial t}
$$

The energy flux, in Watt $/ \mathrm{m}^{2}$, through $A-A$, has the momentary value

$$
I=\left(p-p_{0}\right) v
$$

and its mean value, indicated with the overbar, is

$$
\bar{I}=\overline{\left(p-p_{0}\right) v} \text {. }
$$

The mean can be taken with respect to time, at one location $X$, or with respect to $X$, at one time. When the process is statistically stationary these mean values are identical and both equal to the ensemble average, which is the average over all the possible configurations of a large collection of bubbles like in Fig. 3. Eventually we are interested in the spectral distribution of $I$, which calls for the use of Fourier transforms. Since, however, the Fourier transforms of stochastic variables as $\eta_{2}$ and $\varphi$ do not exist recourse has to be made to either generalised functions or Fourier-Stieltjes transforms. We follow Buist [1] in employing the latter and introduce the Fourier-Stieltjes coefficient $\mathrm{d} \phi$, associated with $\varphi$ by $^{\dagger}$

$$
\varphi(X, y, t)=(2 \pi)^{-1} \int_{\omega} \int_{\kappa} \mathrm{d} \phi(\kappa, \omega, y) \mathrm{e}^{\mathrm{i} \omega t} \mathrm{e}^{\mathrm{i} \kappa X}
$$

Likewise we define the Fourier-Stieltjes coefficient $\mathrm{d} \chi(\kappa, \omega)$ by

$$
\eta_{2}(X, t)=(2 \pi)^{-1} \int_{\omega} \int_{\kappa} \mathrm{d} \chi(\kappa, \omega) \mathrm{e}^{\mathrm{i} \omega t} \mathrm{e}^{\mathrm{i} \kappa X}
$$

Note that $k$ is the wave number $k=2 \pi / \lambda$ of the wavy wall, whereas $\kappa$ is used here as transform variable.

\footnotetext{
${ }^{\dagger}$ The definition of the Fourier or Fourier-Stieltjes transform is chosen here in which there is a factor $(2 \pi)^{-1 / 2}$ in front of the transform with respect to one variable and consequently also in front of the inverse integral. In that case the spectral relations used here, are in accordance with those in the well known book on stochastic phenomena by Stratonovich [8].
} 
The inverse transformations corresponding with (3.9) and (3.10) are

$$
\begin{aligned}
& \frac{\mathrm{d} \phi}{\mathrm{d} \omega \mathrm{d} \kappa}=(2 \pi)^{-1} \iint \varphi(X, y, t) \mathrm{e}^{-\mathrm{i} \omega t} \mathrm{e}^{\mathrm{i} \kappa X} \mathrm{~d} t \mathrm{~d} X, \\
& \frac{\mathrm{d} \chi}{\mathrm{d} \omega \mathrm{d} \kappa}=(2 \pi)^{-1} \iint \eta_{2}(X, t) \mathrm{e}^{-\mathrm{i} \omega t} \mathrm{e}^{\mathrm{i} \kappa X} \mathrm{~d} t \mathrm{~d} X .
\end{aligned}
$$

Using (3.11), (3.12) and the boundary condition (3.4), it follows from transformation of the differential equation (3.5) that

$$
\mathrm{d} \phi(\omega, \kappa, y)=\left[\omega \frac{\exp \left\{-\mathrm{i}(y-H)\left(\omega^{2} / c_{l}^{2}-\kappa^{2}\right)^{1 / 2}\right\}}{\left(\omega^{2} / c_{l}^{2}-\kappa^{2}\right)^{1 / 2}}\right] \mathrm{d} \chi(\omega, \kappa) .
$$

We are interested in the spectral density of the energy flux (3.8). According to the theory of Fourier transforms this is equal to the Fourier transform, or in the present case the Fourier-Stieltjes transform of the cross correlation

$$
\iint\left(p-p_{0}\right)(x, t) v(x+r, t+\tau) \mathrm{d} x \mathrm{~d} t
$$

where $\left(p-p_{0}\right)$ and $v$ are taken at the level $y_{A}$ of the plane $A-A$. In (3.14) the spatial shift $r$ and the time shift $\tau$ are positive, ${ }^{\dagger}$ Taking the Fourier-Stieltjes transform of (3.14) with respect to $r$ and $\tau$ and dividing by $(2 \pi)^{-2}$ gives

$$
\begin{aligned}
& (2 \pi)^{-2} \iint\left(p-p_{0}\right) \exp (\mathrm{i} \omega t) \exp (\mathrm{i} \kappa x) \mathrm{d} x \mathrm{~d} t \iint v(x+r, t+\tau) \\
& \quad \times \exp (-\mathrm{i} \omega(t+\tau)) \exp \{-\mathrm{i} \kappa(x+r)\} \mathrm{d} r \mathrm{~d} \tau
\end{aligned}
$$

Using (3.3), (3.4), (3.6), (3.11) and (3.13), this can be written as

$$
\left\{-\rho_{0} \mathrm{i} \omega^{\prime} \mathrm{d} \phi^{*}\left(\omega^{\prime} \kappa^{\prime}\right) \mathrm{i}\left\{\omega^{2} / c_{l}^{2}-\kappa^{2}\right\}^{1 / 2} \mathrm{~d} \phi(\omega, \kappa)\right\} / \mathrm{d} \omega \mathrm{d} \kappa \mathrm{d} \omega^{\prime} \mathrm{d} \kappa^{\prime}
$$

Here, ${ }^{*}$ denotes a complex conjugate. The product $\mathrm{d} \phi^{*}\left(\omega^{\prime}, \kappa^{\prime}\right) \mathrm{d} \phi(\omega, \kappa)$ is for stationary random processes zero unless $\omega^{\prime}=\omega$ and $\kappa^{\prime}=\kappa$, in which case

$$
\frac{\mathrm{d} \Phi^{*} \mathrm{~d} \Phi}{\mathrm{d} \omega \mathrm{d} \omega^{\prime} \mathrm{d} \kappa \mathrm{d} \kappa^{\prime}}=\Pi_{\varphi} .
$$

$\Pi$ is called the spectral density and the above derivation demonstrates that the spectral density of the emitted sound is the Fourier-Stieltjes transform of the cross correlation (3.14) and, using (3.15) and (3.16) given by

$$
\Psi(\kappa, \omega)=\rho_{0} \omega\left(\omega^{2} / c_{l}^{2}-\kappa^{2}\right)^{1 / 2} \Pi_{\varphi} .
$$

Alternative forms are, when we use (3.13)

${ }^{\dagger}$ Results for negative $r$ and $\tau$ can be obtained by making use of the symmetry properties of the correlation functions. Further $x$ or $X$ may be used, because the difference is an unimportant shift in the spectra. 


$$
\Psi(\kappa, \omega)=\frac{\rho_{0} \omega^{3}}{\left(\omega^{2} / c_{l}^{2}-\kappa^{2}\right)^{1 / 2}} \Pi_{\eta},
$$

where

$$
\mathrm{d} \chi^{*} \mathrm{~d} \chi=\Pi_{\eta} \mathrm{d} \omega \mathrm{d} \kappa
$$

and

$$
\Psi(\kappa, \omega)=\frac{\rho_{0} \omega}{\left(\omega^{2} / c_{l}^{2}-\kappa^{2}\right)^{1 / 2}} \Pi_{v},
$$

where (3.3) and (3.4) are used.

Relation (3.18) is used by Buist [1], whereas we shall in the following section employ the relation (3.20).

\section{Estimate for $\boldsymbol{\Pi}_{v}$}

Referring to Fig. 4, we consider the correlation

$$
v\left(x_{1}, t\right) v\left(x_{1}+r, t+\tau\right)
$$

between the $y$-component of the velocity induced in $x_{1}$ at time $t$ with that induced in $x_{1}+r$ at time $t+\tau$. The mean value of this over all possible $x_{1}$ and $t$ is, with a stationary stochastic process, a function of the shift $r$ and the time shift $\tau$,

$$
C(r, \tau)=\overline{v\left(x_{1}, t\right) v\left(x_{1}+r, t+\tau\right)},
$$

where the mean is indicated with an overbar.

This can be taken as an ensemble average where the ensemble consists of all the possible configurations at time $t$ and at time $t+\tau$. For each configuration bubbles which are close to the point of observation ( $x_{1}$ in Fig. $4^{\mathrm{a}}$ and $x_{1}+r$ in Fig. $4^{\mathrm{b}}$ ) have more influence that those at a larger distance. Those at a distance larger than a multiple of the bubble radius a hardly contribute. Since the probability of finding a bubble centre in a sphere around $x_{1}$ (or $x_{1}+r$ in Fig. $4^{b}$ ) of such a radius, is just the concentration $\alpha$ we can, in an accuracy to order $\alpha$, consider each configuration to consist of only one bubble. This is located in Fig. $4^{a}$ at a distance $R_{1}$ from $x_{1}$ and in Fig. $4^{\mathrm{b}}$ at a distance $R_{2}$ from $x_{1}+r$. If the Fourier-Stieltjes transform of $v$ is indicated with $\mathrm{d} B$, we have, in analogy with (3.14)-(3.17)

$$
\frac{\mathrm{d} B^{*} \mathrm{~d} B}{\mathrm{~d} \omega \mathrm{d} \kappa}=\Pi_{v}=(2 \pi)^{-1} \iint C(r, \tau) \mathrm{e}^{-\mathrm{i} \omega \tau-\mathrm{i} \kappa r} \mathrm{~d} r \mathrm{~d} \tau
$$

$C(r, \tau)$ being defined by (4.2).

We start with calculating the velocity induced in $y$ direction in Fig. $4^{\mathrm{a}}$. The sound problem formulated in Section 1 and Section 2 is two-dimensional, whereas here we have a three-dimensional problem. We employ spherical coordinates $R, \theta$ and $\tilde{\theta}, R$ and $\theta$ being indicated in Fig. $4^{\text {a }}$. The angular coordinate $\tilde{\theta}$ is needed when we have to determine the $y$ 

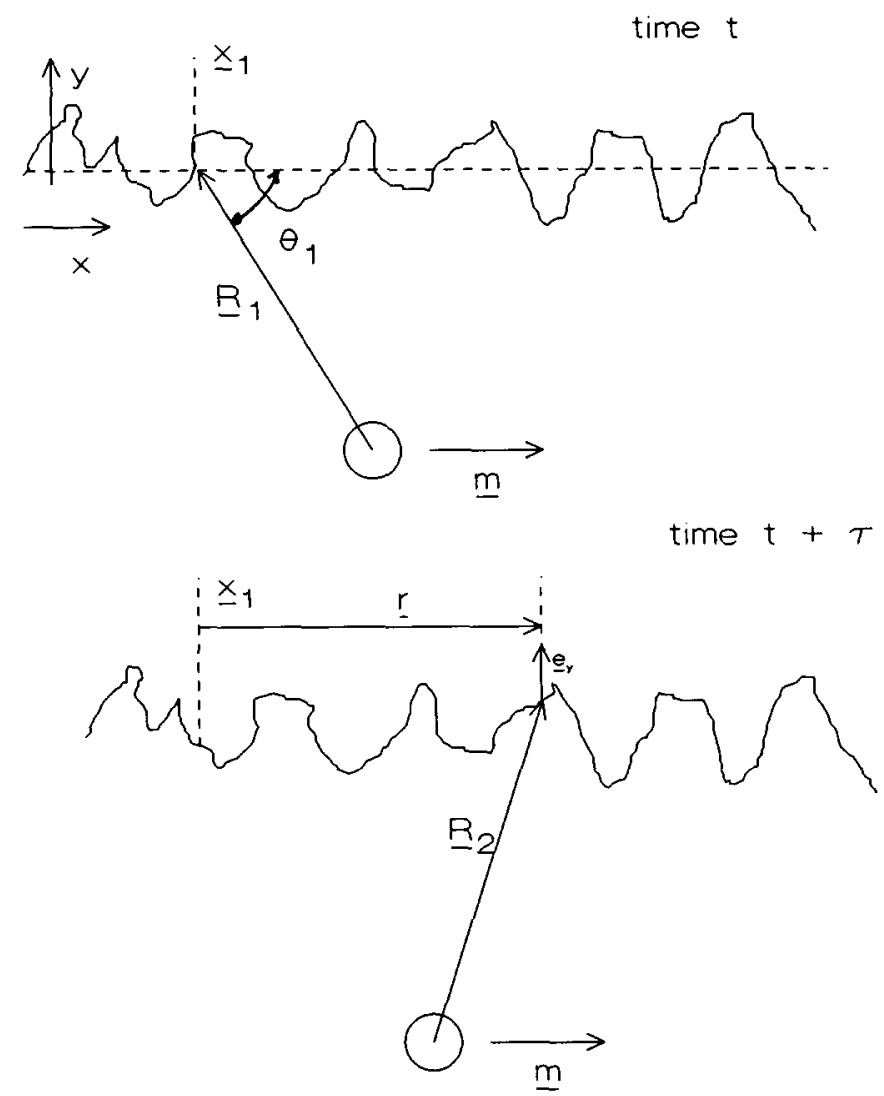

Fig. 4. (a) At time $t$, motion in the point $\mathbf{x}_{1}$ of the interface is induced by a bubble at distance $\mathbf{R}_{1}$ from $\mathbf{x}_{1}$, with dipole strength $\mathbf{m}$. (b) At time $t+\tau$ the influence is considered in a point $x_{1}+r$ of the interface separated from the bubble by a distance $\mathbf{R}_{2}$. The dipole strength of the bubble is $\mathbf{m}$.

component of the velocity induced in $x_{1}$. With constant bubble volume the motion of the bubble is represented with a dipole of strength $\mathbf{m}$ proportional with and in the direction of the momentary relative velocity of the bubble, $\mathbf{q}$, say,

$$
\mathbf{m}=\mathbf{q} a^{3 / 2} \text {. }
$$

The flow potential $\Omega$ is

$$
\Omega=\frac{\mathbf{m} \cdot \mathbf{R}}{R^{3}}
$$

The velocity induced in any point at distance $\mathbf{R}$ from the centre is

$$
\nabla \Omega=\frac{\mathbf{m}}{R^{3}}-3 \frac{\mathbf{m} \cdot \mathbf{R}}{R^{5}} \mathbf{R}
$$

The component of this in $y$-direction is, $\mathbf{e}_{y}$ being the unit vector normal to the interface

$$
v\left(x_{1}, t\right)=\frac{\mathbf{m} \cdot \mathbf{e}_{y}}{R^{3}}-3 \frac{\mathbf{m} \cdot \mathbf{R}}{R^{5}}\left(\mathbf{R} \cdot \mathbf{e}_{y}\right) .
$$


The dipole strength is equal to the instantaneous relative velocity of the bubble under consideration. In Section 2 we have calculated the average, over the mesoscale $l$. The true relative velocity fluctuates around this value. For present purposes it is sufficient to take a dipole strength $\mathbf{m}$ based on the mean velocity, given by (2.9) and (2.10).

The relative velocity is parallel to the surface, so the first term on the right-hand side of (4.7) has no component in $y$-direction. With the coordinates indicated in Fig. $4^{\mathrm{a}}$, the second term in the right-hand side of (4.7) becomes

$$
v\left(x_{1}, R_{1}\right)=\frac{3 m R_{1}^{2} \cos \theta_{1} \sin \theta_{1} \sin \tilde{\theta}_{1}}{R_{1}^{5}}
$$

A similar expression can be written down for the situation sketched in Fig. $4^{\mathrm{b}}$ with $R_{2}, \theta_{2}, \tilde{\theta}_{2}$. Calculating the ensemble average amounts to multiplying the latter expression $v\left(x_{1}+r, R_{2}\right)$ with $v\left(x_{1}, R_{1}\right)$, in (4.8), with the volume element in the half space $y \leqslant 0$ in Fig. $4^{\mathrm{a}}$ and $4^{\mathrm{b}}$ and finally with the probability of finding a bubble centre in $\mathbf{R}_{1}$ at time $t$ and one in $\mathbf{R}_{2}$ at time $t+\tau$. To calculate the ensemble average exactly, one should know more about the fluctuations of the velocity of each bubble, which result from interactions. When these fluctuations are completely random, the configurations at time $t$ and time $t+\tau$ are completely uncorrelated and since the average of both $v\left(x_{1}, R_{1}\right)$ and $v\left(x_{1}+r, R_{2}\right)$ are zero, the contribution to the mean given in (4.2) from times $\tau$, other than zero, will be small. We therefore confine ourselves to the correlation for $\tau=0$ and consider

$$
C(r)=\overline{v\left(x_{1}, t\right) v\left(x_{1}+r, t\right)}
$$

We have seen in (4.3) how the wave number-frequency power spectrum $\Pi_{v}$ is related to $C(r, \tau)$. The inverse of (4.3) is

$$
C(r, \tau)=(2 \pi)^{-1} \iint \Pi_{v}(\omega, \kappa) \mathrm{e}^{\mathrm{i} \omega \tau} \mathrm{e}^{\mathrm{i} \kappa r} \mathrm{~d} \omega \mathrm{d} \kappa
$$

Similarly, putting $\tau=0$

$$
C(r)=(2 \pi)^{-1} \int_{\kappa} \Pi_{v}^{l}(\kappa) \mathrm{e}^{\mathrm{i} \kappa r} \mathrm{~d} \kappa
$$

where

$$
\Pi_{v}^{l}(\kappa)=\int_{\omega} \Pi_{v}(\omega, \kappa) \mathrm{d} \omega
$$

We see that while in $(3.20) \Pi_{v}(\kappa, \omega)$ occurs at the right-hand side, the wave number spectrum associated with $C(r)$ gives us only the integral of $\Pi_{v}$ over all frequencies. However if the main contribution to $C(r, \tau)$ comes from $\tau=0$ as is made plausible above we put, approximately

$$
C(r, \tau)=C(r) \delta(\tau)
$$

and then, from (4.3) 


$$
\begin{aligned}
\Pi_{v} & =(2 \pi)^{-1} \iint C(r) \delta(\tau) \mathrm{e}^{-\mathrm{i} \omega \tau} \mathrm{e}^{\mathrm{i} \kappa r} \mathrm{~d} r \mathrm{~d} \tau \\
& =(2 \pi)^{-1} \int C(r) \mathrm{e}^{-\mathrm{i} \kappa r} \mathrm{~d} r
\end{aligned}
$$

which is the inverse of (4.10).

In other words, the (reasonable) assumption that the mean contribution to $C(r, \tau)$ comes from the autocorrelation $C(r, 0)$ enables us to determine $\Pi_{v}$, which we need in order to evaluate $\Psi(\kappa, \omega)$ in $(3.20)$.

If we take $\tau=0$, we have to consider the situation in Fig. 5. Taking $\theta_{1}=\theta_{2}=\theta$ etc., we have to formulate the ensemble average (4.9). The probability of finding a bubble centre in the element of volume $R^{2} \sin \theta \mathrm{d} R \mathrm{~d} \theta \mathrm{d} \tilde{\theta}$ is

$$
n R^{2} \sin \theta \mathrm{d} R \mathrm{~d} \theta \mathrm{d} \tilde{\theta}
$$

where $n$ is the number density. Using (4.8) for $v\left(x_{1}, R\right)$ and a similar expression for $v\left(x_{1}+r, R\right)$ we have

$$
C(r)=-\frac{1}{2} \pi n \int_{0}^{\pi} \mathrm{d} \theta \int_{a}^{\infty} \frac{9 m^{2}(R \cos \theta-r) \cos \theta \sin ^{3} \theta \mathrm{d} R \mathrm{~d} \theta}{\left(R^{2}+r^{2}-2 R r \cos \theta\right)^{5 / 2}} .
$$

The factor $\pi / 2$ in front of the integrals in (4.13) is from integration of $\sin ^{2} \tilde{\theta}$ (resulting from $\sin \tilde{\theta}$ in the expression for $v\left(x_{1}, R\right)$ and another one in the expression for $\left.v\left(x_{1}+r, R\right)\right)$ over the interval from 0 to $\pi$. Since the bubbles have a non zero radius, $a$, and the point $x_{1}$ must be in liquid, $R$ runs from $a$ to $\infty$. The spatial shift $r$ is positive. Carrying out the integration over $R$ and writing

$$
s=\cos \theta
$$

gives

$$
\begin{aligned}
C(r)= & 3 / 2 \pi m^{2} n\left[\int_{-1}^{1}\left\{2-\frac{2(a-s r)}{\left(r^{2}+a^{2}-2 s a r\right)^{1 / 2}}-\frac{(a-s r)\left(1-s^{2}\right) r^{2}}{\left(r^{2}+a^{2}-2 s a r\right)^{3 / 2}}\right\} \frac{s \mathrm{~d} s}{r^{3}}\right. \\
& \left.-\int_{-1}^{1} \frac{s^{2}\left(1-s^{2}\right) \mathrm{d} s}{\left(r^{2}+a^{2}-2 s a r\right)^{3 / 2}}\right] .
\end{aligned}
$$

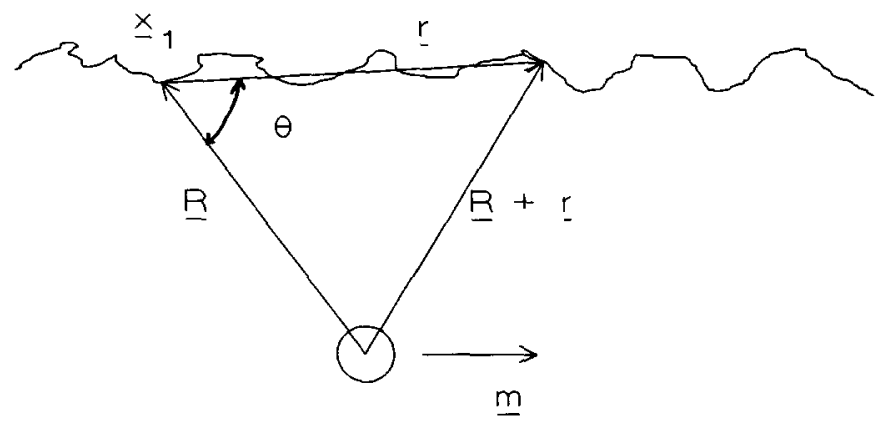

Fig. 5. Scheme for the calculation of the cross correlation between the velocity perturbation in $\mathbf{x}_{1}$ and $\mathbf{x}_{1}+\mathbf{r}$ caused by a bubble moving relative to the fluid with dipole strength $\mathbf{m}$. 
Evaluation of the integrals in (4.15), for details see the Appendix, results in

$$
C(r)=3 \pi m^{2} n\left(\frac{2}{3} \frac{1}{r^{3}}-\frac{4}{5} \frac{a^{2}}{r^{5}}\right)
$$

If there is a bubble centre in $x_{1}+a$, the point nearest to $x_{1}$ with which correlation can be made, is $x_{1}+2 a$. Therefore $r$ runs from $2 a$ to $\infty$. With use of (2.1) and (4.4) we write

$$
C(r)=3 / 8 \alpha q^{2} a^{3}\left(\frac{1}{r^{3}}-\frac{6}{5} \frac{a^{2}}{r^{5}}\right), \quad r>2 a
$$

The correlation function $C(r)$ tends to zero for $r \rightarrow \infty$, as it should, but rather slowly, like $r^{-3}$. It is interesting to note that the decay of $C(r)$ with $r$ is algebraic rather than the exponential behaviour which is often encountered in turbulence. The wavenumber spectrum is now obtained by taking the Fourier transform of (4.17) with respect to $r$,

$$
\Pi_{v}^{\prime}(\kappa)=2(2 \pi)^{-1} \int_{2 a}^{\infty} C(r) \cos (\kappa r) \mathrm{d} r
$$

Introducing (4.17) into (4.18) and evaluating the integrals gives

$$
\Pi_{v}^{\prime}(\kappa)=\frac{3 / 4 \alpha a^{3} q^{2}}{2 \pi}\left\{0.11 a^{-2}+O\left(a^{2} \kappa^{2}\right) a^{-2}\right\} .
$$

The value of $\Pi_{v}^{\prime}$ can be determined to any accuracy in $(\kappa a)$. We need only the first term between brackets of (4.19), for the following reason. As follows from (3.20), for given $\omega, \kappa$ runs from 0 to $\omega / c_{l}$. Hence the maximum value of $\kappa a$ is $\omega a / c_{l}$. With $\omega \sim 10^{3}, a \sim 10^{-3}$ and $c_{l}=1500 \mathrm{~m} / \mathrm{s}$ this is small enough to neglect terms in $(\kappa a)^{2}$.

\section{Estimate for radiated sound intensity produced by relative motion}

Rather than looking at the wavenumber-frequency spectral density, we consider the total emitted sound intensity $\int \psi \mathrm{d} \omega \mathrm{d} \kappa$. We have found in the previous section that to the second order in $(\kappa a), \Pi_{v}^{\prime}$ does not depend on $\kappa$.

Hence, carrying out the integration over $\kappa$, we have from (3.19)

$$
\int \psi(\omega, \kappa) \mathrm{d} \omega \mathrm{d} \kappa=\bar{\omega} \rho_{0} \int_{0}^{\omega / c_{l}} \frac{\Pi_{v}^{\prime}(\kappa) \mathrm{d} \kappa}{\left(\omega^{2} / c_{l}^{2}-\kappa^{2}\right)^{1 / 2}} .
$$

Here $\bar{\omega}$ is some representative frequency, for example at the centre of gravity of the spectral density distribution. Taking now for $\Pi_{v}^{\prime}(\kappa)$ the zeroth order term on the right-hand side of (4.19) we obtain

$$
\frac{1}{\bar{\omega}} \int \psi(\omega, \kappa) \mathrm{d} \omega \mathrm{d} \kappa=\left(2.1 \times 10^{-2}\right) \rho_{0} \alpha a q^{2},
$$

as an estimate of the sound intensity emitted in a unit element along the frequency axis. The magnitude of this, of course, depends on many parameters. As an example, take $\rho_{0}=10^{3} \mathrm{~kg} /$ $\mathrm{m}^{3}, \alpha=10^{-2}, a=10^{-3} \mathrm{~m}$. The value of $q$ is of the order $2 \varepsilon k U_{\infty}$, when we base ourselves on 
the case where inertia forces dominate over viscous forces in the relative motion. The value of $U_{\infty}$ will be close to $10 \mathrm{~m} / \mathrm{s}$ under cavitating circumstances. For $\varepsilon k=2 \pi \varepsilon / \lambda$ we take $2 \pi \times 0.1=0.2 \pi$. With these values the right-hand side of $(4.20)$ is $83 \times 10^{-4}$. With $\varepsilon k=$ $2 \pi \times 10^{-2}$, and the other values unchanged, this is $0.83 \times 10^{-4}$.

If we express this in $\mathrm{dB}$, defined as

$$
10 \log _{10} \text { (intensity) }+120
$$

we obtain $79 \mathrm{~dB}$ in the second case and $99 \mathrm{~dB}$ in the first case. The intensity is here in watt sec per unit length in $x$ direction. This can be compared with the estimate of $90 \mathrm{~dB}$ given in Buist [1] due to fluctuations in void fraction due to pressure fluctuations. It turns out that relative motion gives contributions of equal magnitude.

\section{Conclusion}

In this paper it has been investigated what the sound emission is from a bubble-liquid layer into pure water, when attention is focused on relative motion between bubbles and liquid. It turns out that the intensity of the emitted sound is comparable to that produced by void fraction fluctuations of other nature such as investigated in the thesis of Buist [1]. Finally it should be noted that the present investigation is of interest not only in the context of cavitation sound in which it is considered here. It can be applied also in studies of flow noise whenever relative motion is important. A possible further extension could be the relative motion due to turbulence. Such a study would be complementary to Crighton \& Ffowcs Williams [9], where the sound emission of a turbulent bubbly fluid is described caused by monopole radiation.

\section{Appendix}

The first and the third integral in the square brackets in (4.13) give together

$$
\frac{a}{r} \int_{-1}^{1} \frac{\left(1-s^{2}\right) s \mathrm{~d} s}{\left\{r^{2}+a^{2}-2 s a r\right\}^{3 / 2}}
$$

whence

$$
C(r)=-\frac{3}{2} \pi m^{2} n\left[\frac{a}{r} \int_{-1}^{1} \frac{\left(1-s^{2}\right) s \mathrm{~d} s}{\left\{r^{2}+a^{2}-2 s a r\right\}^{3 / 2}}-2 \int_{-1}^{1} \frac{s \mathrm{~d} s}{r^{3}}+\frac{2}{r^{3}} \int_{-1}^{1} \frac{(a-s r) s \mathrm{~d} s}{\left\{r^{2}+a^{2}-2 s a r\right\}^{1 / 2}}\right] .
$$

Now define

$$
I_{i j}=\int_{-1}^{1} \frac{s^{i} \mathrm{~d} s}{\left\{r^{2}+a^{2}-2 s a r\right\}^{j / 2}},
$$

then

$$
C(r)=-\frac{3}{2} \pi m^{2} n\left[\frac{a}{r}\left(I_{13}-I_{33}\right)+\frac{2 a}{r^{3}} I_{11}-\frac{2}{r^{2}} J_{21}\right] .
$$


Evaluation of the integrals gives

$$
\begin{aligned}
& I_{13}=\frac{2 a}{r^{2}\left(r^{2}-a^{2}\right)} ; \quad I_{33}=\frac{2}{a\left(r^{2}-a^{2}\right)}-\frac{2}{a r^{2}}-\frac{4 a}{5 r^{4}} ; \\
& I_{11}=\frac{2 a}{3 r^{2}} ; \quad I_{21}=\frac{2}{3 r}+\frac{4 a^{2}}{15 r^{3}} .
\end{aligned}
$$

Introducing these values in the expression for $C(r)$ results in (4.14) in the main text.

\section{References}

1. J. Buist, On the origin and acoustical behaviour of cloud cavitation. Thesis, University of Twente (1991).

2. R. Omta, Oscillations of a cloud of bubbles of small and not so small amplitude. J. Acoust. Soc. Am. 82 (1987) 1018-1033.

3. J. Buist, Acoustics of travelling bubbles cavitation. In: G.E.A. Meier and P.A. Thompson (eds), Proc. IUTAM Symp. on Adiabatic Waves in Liquid-Vapor Systems. Springer (1990) pp. 351-360.

4. A. Biesheuvel and L. van Wijngaarden, Two-phase flow equations for a dilute dispersion of gas bubbles in liquid. J. Fluid Mech. 148 (1984) 304-318.

5. L. d'Agostino and C.E. Brennen, Linearized dynamics of two-dimensional bubbly and cavitating flows over slender surfaces. J. Fluid Mechanics 192 (1988) 485-509.

6. L. van Wijngaarden, One-dimensional flows of liquids containing small gas bubbles. Ann. Rev. Fluid Mech. 4 (1972) 369-396.

7. H.W. Liepmann and A. Roshko, An Introduction in Gas Dynamics. Wiley \& Sons (1958).

8. R.L. Stratonovich, Topics in the Theory of Random Noise, Vol. I. Gordon \& Breach (1963).

9. D.G. Crighton and J.E. Ffowcs Williams, Sound generation by turbulent two-phase flow. J. Fluid Mech. 36 (1969) 585-603. 